\title{
Adaptive rational interpolation: Arnoldi and Lanczos-like
}

\section{equations}

\author{
Michalis Frangos* Imad M. Jaimoukha ${ }^{\dagger}$
}

Received 13 June 2007; Accepted June 2008, European Journal of Control (2008)4:342-354

(C) 2008 EUCA DOI:10.3166/EJC.14.342354

\begin{abstract}
The Arnoldi and Lanczos algorithms, which belong to the class of Krylov subspace methods, are increasingly used for model reduction of large scale systems. The standard versions of the algorithms tend to create reduced order models that poorly approximate low frequency dynamics. Rational Arnoldi and Lanczos algorithms produce reduced models that approximate dynamics at various frequencies. This paper tackles the issue of developing simple Arnoldi and Lanczos equations for the rational case. This allows a simple error analysis to be carried out for both algorithms and permits the development of computationally efficient model reduction algorithms, where the frequencies at which the dynamics are to be matched can be updated adaptively.
\end{abstract}

\section{Keywords}

Model reduction, Krylov subspaces, Arnoldi algorithm, Lanczos algorithm, rational interpolation, adaptive interpolation

\footnotetext{
${ }^{*}$ Department of Electrical and Electronic Engineering, Imperial College London, Exhibition Road, SW7 2AZ, UK (frangos@mit.edu; michalis.frangos99@imperial.ac.uk).

${ }^{\dagger}$ Department of Electrical and Electronic Engineering, Imperial College London, Exhibition Road, London, SW7 2AZ, UK (i.jaimouka@imperial.ac.uk).
} 


\section{Introduction}

Consider a linear time-invariant single-input single-output system described by the equations

$$
E \dot{x}(t)=A x(t)+B u(t), \quad y(t)=C x(t)
$$

where $x(t) \in \mathbb{R}^{n}$ denotes the state vector and $u(t)$ and $y(t)$ the scalar input and output signals, respectively. The system matrices $A, E \in \mathbb{R}^{n \times n}$ are assumed to be large and sparse, and $B, C^{\prime} \in \mathbb{R}^{n}$. These assumptions are met by large scale models in many applications. The transfer function for the system in (1) is denoted as $G(s)=C(s E-A)^{-1} B$. To simplify subsequent analysis and design based on the large $n$th order model in (1), the model reduction problem seeks an approximate $m$ th order model of the form $G_{m}(s)=C_{m}\left(s E_{m}-A_{m}\right)^{-1} B_{m}$ where $E_{m}, A_{m} \in \mathbb{R}^{m \times m}, B_{m}, C_{m}^{\prime} \in \mathbb{R}^{m}$ and $m<n$. We also assume that $E$ and $E_{m}$ are non-singular.

Unlike many existing model reduction methods such as balanced truncation and Hankel norm approximations [2,39], Krylov projection methods, and in particular the Arnoldi and Lanczos algorithms $[4,7,26,27,36]$, exploit the sparsity of the large scale model and have been extensively used for model reduction of large scale systems; see $[3,8,36]$ and the references therein. In these approaches, $G_{m}(s)$ is computed such that it matches the moments of $G(s)$, that is the value of $G(s)$ and its derivatives, at certain interpolation points. In the standard approaches, the moments are matched at $\infty[3,16]$. The problem of interpolating around infinity is known in the literature as the partial realisation problem [19]. This has the advantage that, in the case that $E=I_{n}$, only matrix-vector products are needed in calculations involving the large matrix A. Furthermore, a set of equations known as the Arnoldi or Lanczos equations are satisfied and are useful for error analysis and restarts $[22,24,25,30,37]$. In [12] the Arnoldi and Lanczos equations are given for the two-sided Arnoldi and non-symmetric Lanczos algorithms, which are algorithms that produce Padé models [8] by interpolating the original system around infinity. A survey on Krylov space methods based on single point intepolating approximations can be found in $[6]$ and the references therein.

The disadvantage is that single point interpolating approximations tend to approximate $G(s)$ poorly at other frequencies unless the order of the approximation is increased considerably or certain types of restarts are introduced [30].

To ameliorate this problem rational Arnoldi and Lanczos algorithms [17, 21,32-34] have been developed which produce reduced models that match the moments of $G(s)$ at different frequencies. Notwithstanding the greatly improved approximation offered by the rational Arnoldi and Lanczos techniques, there are some outstanding issues that need to be addressed and are summarized below. 
1. No simple Arnoldi- and Lanczos-like equations have been derived in the literature in the rational case. The authors in $[17,18,28,34]$ derive equations that describe the rational algorithms; however these are not in the standard Arnoldi and Lanczos form.

2. No error analysis for the rational case has been derived comparable to the standard algorithms. This is important for deriving residual error expressions, perturbation analysis and for best choice of the interpolation frequencies.

The contribution of this paper is in addressing in detail each of these issues which were briefly described in [14]. Section 2 gives a review of approximation techniques by moment matching. The standard Arnoldi and the rational Arnoldi algorithms for moment matching are also described. In section 3 Arnoldi- and Lanczos-like equations for the rational case are derived. In section 4 the Arnoldi and Lanczos equations are used to give an error analysis for the rational case. Simple residual error expressions are derived and norm-bounded as well as linear fractional transformation perturbation analysis is given. In section 5 adaptive schemes based on the error analysis are described. Section 6 gives some numerical results to illustrate the adaptive methods developed in this paper. Finally section 7 gives our conclusions.

\section{Krylov based methods for model reduction}

\subsection{Moment matching problem}

The system in (1) can be expanded by Taylor series around an interpolation point $s_{0} \in \mathbb{C}$ as

$$
G(s)=\mu_{0}+\mu_{1}\left(s-s_{0}\right)+\mu_{2}\left(s-s_{0}\right)^{2}+\cdots
$$

where the Taylor coefficients $\mu_{i}$ are known as the moments of the system around $s_{0}$ and are related to the transfer function of the system and its derivatives evaluated at $s_{0}$. The approximation problem by moment matching is to find a lower order system $G_{m}(s)$ with transfer function expanded as

$$
G_{m}(s)=\hat{\mu}_{0}+\hat{\mu}_{1}\left(s-s_{0}\right)+\hat{\mu}_{2}\left(s-s_{0}\right)^{2}+\cdots
$$

such that $\mu_{i}=\hat{\mu}_{i}$, for $i=0,1, \ldots, m$.

To simplify the presentation of our results, we only consider the case when $E=I_{n}$ and we will write $G(s)=C\left(s I_{n}-A\right)^{-1} B \stackrel{s}{=}(A, B, C, 0)$ or

$$
G(s) \stackrel{s}{=}\left[\begin{array}{c|c}
A & B \\
\hline C & 0
\end{array}\right] .
$$


In the case where $s_{0}=\infty$ the moments are called Markov parameters and are given by $\mu_{i}=C A^{i} B$. The moments around an arbitrary interpolation point $s_{0} \in \mathbb{C}$ are known as shifted moments and they are defined as $\mu_{i}=C\left(s_{0} I_{n}-A\right)^{-i} B$.

A more general definition of approximation by moment matching is related to rational interpolation. By rational interpolation we mean that the reduced order system matches the moments of the original system at multiple interpolation points.

Let $V_{m}, W_{m} \in \mathbb{C}^{n \times m}$. By projecting the states of the high order system with the projector

$$
P_{m}=V_{m}\left(W_{m}^{\prime} V_{m}\right)^{-1} W_{m}^{\prime}
$$

assuming that $E_{m}=W_{m}^{\prime} V_{m}$ is non-singular, a reduced order model is obtained as:

$$
G_{m}(s) \stackrel{s}{=}\left[\begin{array}{c|c}
E_{m}^{-1} A_{m} & E_{m}^{-1} B_{m} \\
\hline C_{m} & 0
\end{array}\right]:=\left[\begin{array}{c|c}
\left(W_{m}^{\prime} V_{m}\right)^{-1} W_{m}^{\prime} A V_{m} & \left(W_{m}^{\prime} V_{m}\right)^{-1} W_{m}^{\prime} B \\
\hline C V_{m} & 0
\end{array}\right] .
$$

A careful selection of $V_{m}$ and $W_{m}$ as the bases of certain Krylov subspaces results in moment matching. For $A \in \mathbb{C}^{n \times n}, B \in \mathbb{C}^{n}, s \in \mathbb{C}$ and integer $m>0$ a Krylov subspace $\mathcal{K}_{m}(A, B, s)$ is defined as

$$
\begin{aligned}
& \mathcal{K}_{m}(A, B, s):=\operatorname{colsp}\left[\left(s I_{n}-A\right)^{-1} B,\left(s I_{n}-A\right)^{-2} B, \ldots,\left(s I_{n}-A\right)^{-m} B\right], \quad \text { if } s \neq \infty \\
& \mathcal{K}_{m}(A, B, s):=\operatorname{colsp}\left[B, A B, \ldots, A^{(m-1)} B\right], \quad \text { if } s=\infty
\end{aligned}
$$

where colsp denotes column span. If $m=0, \mathcal{K}_{m}(A, B, s)$ is defined to be the empty set.

\subsection{Standard Arnoldi methods}

An iterative method for model reduction based on Krylov projections is the Arnoldi process. The two-sided Arnoldi process, given in Algorithm 1, constructs the bases $V_{m}=\left[v_{1}, \ldots, v_{m}\right] \in \mathbb{C}^{n \times m}$ and $W_{m}=\left[w_{1}, \ldots, w_{m}\right] \in \mathbb{C}^{n \times m}$ for the Krylov subspaces $\mathcal{K}_{m}(A, B, \infty)$ and $\mathcal{K}_{m}\left(A^{\prime}, C^{\prime}, \infty\right)$, respectively, such that they are orthonormal, i.e., $V_{m}^{\prime} V_{m}=W_{m}^{\prime} W_{m}=I_{m}$. We assume that no breakdown (or near breakdown) occurs in Algorithm 1 so that $\left\|\hat{v}_{j}\right\|>\epsilon$ and $\left\|\hat{w}_{j}\right\|>\epsilon$, where $\epsilon$ is a tolerance level, for $j=1, \ldots, m$ and $W_{m}^{\prime} V_{m}$ is non-singular. See [35] for more details on the breakdown of the Arnoldi algorithm. The following equations,

$$
\begin{aligned}
A V_{m} & =V_{m} H_{m}+v_{m+1} C_{V_{m}}, & B & =V_{m} L_{m} \\
W_{m}^{\prime} A & =F_{m} W_{m}^{\prime}+B_{W_{m}} w_{m+1}^{\prime}, & C & =K_{m} W_{m}^{\prime}
\end{aligned}
$$

referred to as the Arnoldi equations hold $[23,25]$, where

$$
\begin{aligned}
& H_{m}=V_{m}^{\prime} A V_{m}, \quad C_{V_{m}}=v_{m+1}^{\prime} A V_{m}, \quad L_{m}=V_{m}^{\prime} B, \\
& F_{m}=W_{m}^{\prime} A W_{m}, \quad B_{W_{m}}=W_{m}^{\prime} A w_{m+1}, \quad K_{m}=C W_{m} \text {. }
\end{aligned}
$$


Pre-multiplying the first set of the Arnoldi equations by $W_{m}^{\prime}$ and solving with respect to $H_{m}$ and $L_{m}$ we have that $H_{m}=E_{m}^{-1} A_{m}-E_{m}^{-1} W_{m}^{\prime} v_{m+1} C_{V_{m}}$ and $L_{m}=E_{m}^{-1} B$. Similarly postmultiplying the second set of the Arnoldi equations by $V_{m}$ and rearranging results in $F_{m}=$ $A_{m} E_{m}^{-1}-B_{W_{m}} w_{m+1}^{\prime} V_{m} E_{m}^{-1}$ and $K_{m}=C_{m} E_{m}^{-1}$, respectively. The standard Arnoldi equations can then be transformed to the following set of equations

$$
\begin{aligned}
A V_{m} & =V_{m} E_{m}^{-1} A_{m}+\left(I_{n}-P_{m}\right) v_{m+1} C_{V_{m}}, & B & =V_{m} E_{m}^{-1} B_{m} \\
W_{m}^{\prime} A & =A_{m} E_{m}^{-1} W_{m}^{\prime}+B_{W_{m}} w_{m+1}^{\prime}\left(I_{n}-P_{m}\right), & C & =C_{m} E_{m}^{-1} W_{m}^{\prime}
\end{aligned}
$$

where $E_{m}, A_{m}, B_{m}$ and $C_{m}$ are defined in (3) and where $P_{m}$ is defined in (2).

The bases $V_{m}$ and $W_{m}$ are constructed such that $\mathcal{K}_{m}(A, B, \infty) \subseteq \operatorname{colsp}\left(V_{m}\right)$ and $\mathcal{K}_{m}\left(A^{\prime}, C^{\prime}, \infty\right) \subseteq$ $\operatorname{colsp}\left(W_{m}\right)$ and so the reduced order system $G_{m}(s)$, defined in (3), matches the first $2 m$ Markov parameters of $G(s)$ [18]. The approximation forward error $\epsilon(s):=G(s)-G_{m}(s)$ can be shown

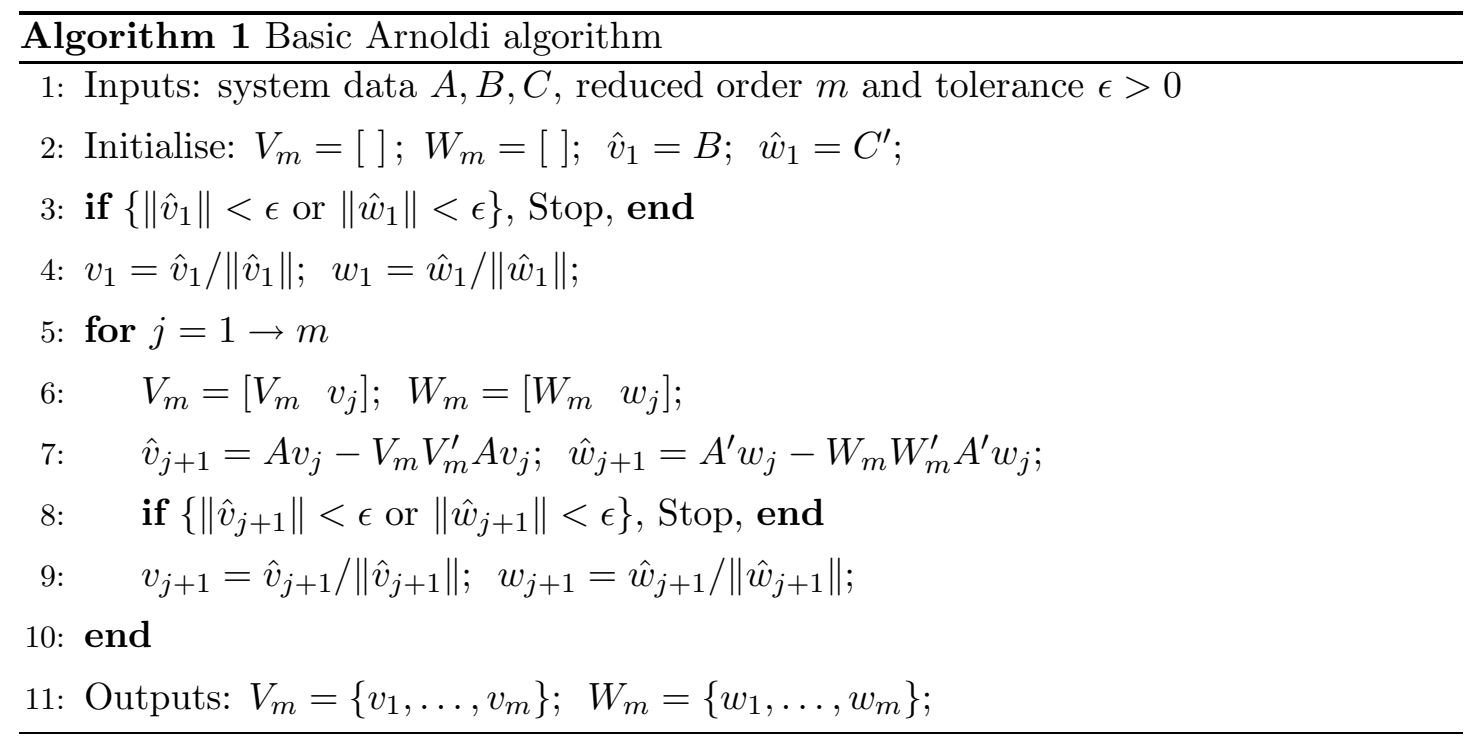

to be

$$
\epsilon(s)=R_{C}(s)^{\prime}\left(s I_{n}-A\right)^{-1} R_{B}(s),
$$

where $R_{C}(s)$ and $R_{B}(s)$, known as the residual errors, are given by

$$
R_{B}(s)=B-\left(s I_{n}-A\right) V_{m} X_{m}(s), \quad R_{C}(s)=C^{\prime}-\left(s I_{n}-A\right)^{\prime} W_{m} Y_{m}(s),
$$

and where $Y_{m}(s)$ and $X_{m}(s)$ are the solutions of the system of equations

$$
\left(s E_{m}-A_{m}\right) X_{m}(s)=B_{m}, \quad\left(s E_{m}-A_{m}\right)^{\prime} Y_{m}(s)=C_{m}^{\prime}
$$

and satisfy the Petrov-Galerkin conditions $W_{m}^{\prime} R_{B}(s)=V_{m}^{\prime} R_{C}(s)=0$. 
Using the Arnoldi equations, the expressions for the residual errors can be simplified as

$$
\begin{aligned}
R_{B}(s) & =\left(I_{n}-P_{m}\right) v_{m+1} C_{V_{m}}\left(s E_{m}-A_{m}\right)^{-1} B_{m}=v_{m+1} C_{V_{m}}\left(s I_{m}-H_{m}\right)^{-1} L_{m} \\
R_{C}(s)^{\prime} & =C_{m}\left(s E_{m}-A_{m}\right)^{-1} B_{W_{m}} w_{m+1}^{\prime}\left(I_{n}-P_{m}\right)=K_{m}\left(s I_{m}-F_{m}\right)^{-1} B_{W_{m}} w_{m+1}^{\prime}
\end{aligned}
$$

which involve terms related to the reduced order system only.

\subsection{The rational Arnoldi method}

The rational Arnoldi procedure [32-34] is an algorithm for constructing orthonormal bases of the union of Krylov subspaces. Let $V_{m}, W_{m} \in \mathbb{C}^{n \times m}$ be the bases of such subspaces and let $P_{m}$ be a projector defined as $P_{m}=V_{m}\left(W_{m}^{\prime} V_{m}\right)^{-1} W_{m}^{\prime}$. Applying this projector on the system in (1) a reduced order system is obtained with a transfer function as in (3). The next result shows that a proper selection of Krylov subspaces will result in reduced order systems that matches the moments of the system at given interpolation frequencies.

Theorem 2.1. [18] Let $S=\left\{s_{1}, s_{2}, \ldots, s_{K}\right\} \subset \mathbb{C}$ and $\tilde{S}=\left\{\tilde{s}_{1}, \tilde{s}_{2}, \ldots, \tilde{s}_{\tilde{K}}\right\} \subset \mathbb{C}$ be two sets of distinct interpolation points, with multiplicities $m_{s_{1}}, m_{s_{2}}, \ldots, m_{s_{K}}$, and $\tilde{m}_{s_{1}}, \tilde{m}_{s_{2}}, \ldots, \tilde{m}_{s_{\tilde{K}}}$, respectively. Suppose that $V_{m}, W_{m} \in \mathbb{C}^{n \times m}$ satisfy

$$
\begin{aligned}
& \operatorname{colsp}\left(V_{m}\right) \supseteq \mathcal{K}_{m_{s_{1}}}\left(A, B, s_{1}\right) \cup \cdots \cup \mathcal{K}_{m_{s_{K}}}\left(A, B, s_{K}\right) \\
& \operatorname{colsp}\left(W_{m}\right) \supseteq \mathcal{K}_{m_{\tilde{s}_{1}}}\left(A^{\prime}, C^{\prime}, \tilde{s}_{1}^{\prime}\right) \cup \cdots \cup \mathcal{K}_{m_{\tilde{s}_{K}}}\left(A^{\prime}, C^{\prime}, \tilde{s}_{\tilde{K}^{\prime}}^{\prime}\right)
\end{aligned}
$$

where $\sum_{k=1}^{K} m_{s_{k}}=\sum_{k=1}^{\tilde{K}} \tilde{m}_{s_{k}}=m$. Then, assuming that $\left(s I_{n}-A\right)^{-1}$ exists for all $s \in S \cup \tilde{S}$,

- if $s_{i}=\tilde{s}_{j}, G_{m}(s)$ matches the first $m_{s_{i}}+\tilde{m}_{\tilde{s}_{j}}$ moments of $G(s)$ at $s_{i}$

- if $s_{i} \neq \tilde{s}_{j}, G_{m}(s)$ matches the first $m_{s_{i}}$ moments of $G(s)$ at $s_{k}$ and the first $\tilde{m}_{\tilde{s}_{j}}$ moments of $G(s)$ at $\tilde{s}_{k}$, respectively.

The rational Arnoldi algorithm is given in Algorithm 2. For simplicity of presentation we assume that $m_{s_{k}}=m_{\tilde{s}_{k}}$ and also it is assumed that $s_{i} \neq s_{j}$ and $\tilde{s}_{i} \neq \tilde{s}_{j}$ for $i \neq j$.

\section{Arnoldi-like equations in the rational case}

Many of the results based on Krylov subspaces follow from the Arnoldi and Lanczos equations. A main contribution of this work is to derive, with minimum additional effort, the corresponding equations for the rational version of these algorithms $[14,15]$.

The following result derives such a set of equations for the Arnoldi process. 


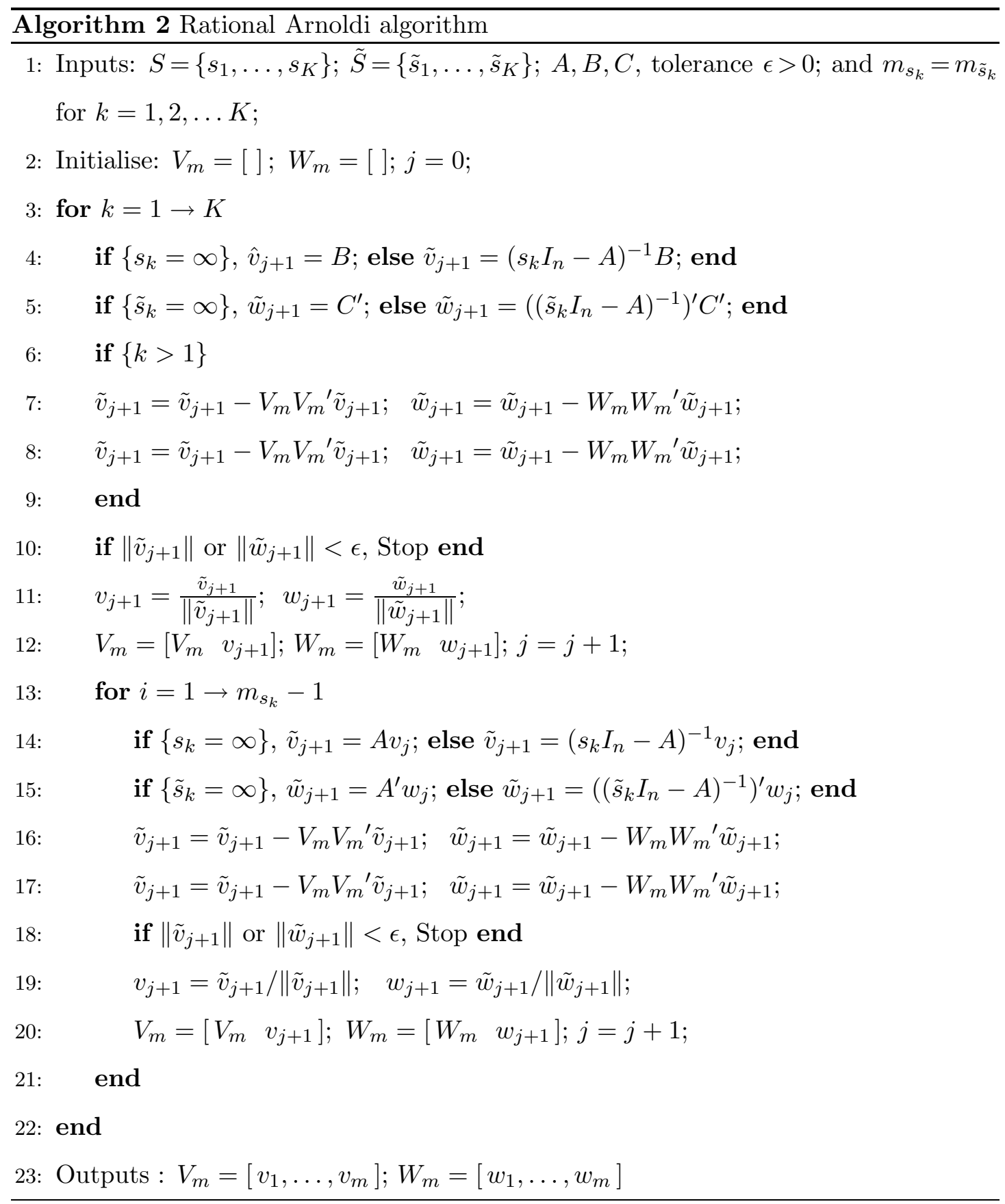


Lemma 3.1. Let all variables be as defined in Theorem 2.1 and let $V_{m}$ and $W_{m}$ be as constructed by Algorithm 2 so that $V_{m}^{\prime} V_{m}=W_{m}^{\prime} W_{m}=I_{m}$. Let $m_{\infty}$ and $\tilde{m}_{\infty}$ be the multiplicities of $\infty$ in $S$ and $\tilde{S}$, respectively $\left(m_{\infty}=0\right.$ if $\infty \notin S$ and $\tilde{m}_{\infty}=0$ if $\left.\infty \notin \tilde{S}\right)$. Define $v_{m+1}, w_{m+1} \in \mathbb{C}^{n}$ such that, with $V_{m+1}:=\left[\begin{array}{ll}V_{m} & v_{m+1}\end{array}\right]$ and $W_{m+1}:=\left[\begin{array}{ll}W_{m} & w_{m+1}\end{array}\right]$,

$$
\begin{aligned}
\operatorname{colsp}\left(\left[\begin{array}{ll}
V_{m} & A^{m_{\infty} B}
\end{array}\right]\right) & =\operatorname{colsp}\left(V_{m+1}\right), & V_{m+1}^{\prime} V_{m+1} & =I_{m+1}, \\
\operatorname{colsp}\left(\left[\begin{array}{cc}
W_{m} & \left(A^{\prime}\right)^{\tilde{m}_{\infty}} C^{\prime}
\end{array}\right]\right) & =\operatorname{colsp}\left(W_{m+1}\right), & W_{m+1}^{\prime} W_{m+1} & =I_{m+1} .
\end{aligned}
$$

1. Define

$\left[\begin{array}{cc}H_{m} & L_{m} \\ C_{V_{m}} & b_{m}\end{array}\right]:=\left[\begin{array}{cc}V_{m}^{\prime} A V_{m} & V_{m}^{\prime} B \\ v_{m+1}^{\prime} A V_{m} & v_{m+1}^{\prime} B\end{array}\right],\left[\begin{array}{cc}F_{m} & B_{W_{m}} \\ K_{m} & c_{m}\end{array}\right]:=\left[\begin{array}{cc}W_{m}^{\prime} A W_{m} & W_{m}^{\prime} A w_{m+1} \\ C W_{m} & C w_{m+1}\end{array}\right]$

Then

$$
\begin{aligned}
A V_{m} & =V_{m} H_{m}+v_{m+1} C_{V_{m}}, & B & =V_{m} L_{m}+v_{m+1} b_{m}, \\
W_{m}^{\prime} A & =F_{m} W_{m}^{\prime}+B_{W_{m}} w_{m+1}^{\prime}, & C & =K_{m} W_{m}^{\prime}+c_{m} w_{m+1}^{\prime} .
\end{aligned}
$$

Furthermore, $b_{m}=0$ if $\infty \in S$ and $c_{m}=0$ if $\infty \in \tilde{S}$.

2. Let $P_{m}, E_{m}, A_{m}, B_{m}, C_{m}, C_{V_{m}}$ and $B_{W_{m}}$ be as defined in (2), (3) and (4). Then

$$
\begin{array}{cc}
A V_{m}=V_{m} E_{m}^{-1} A_{m}+\left(I_{n}-P_{m}\right) v_{m+1} C_{V_{m}}, & B=V_{m} E_{m}^{-1} B_{m}+\left(I_{n}-P_{m}\right) v_{m+1} b_{m}, \\
W_{m}^{\prime} A=A_{m} E_{m}^{-1} W_{m}^{\prime}+B_{W_{m}} w_{m+1}^{\prime}\left(I_{n}-P_{m}\right), & C=C_{m} E_{m}^{-1} W_{m}^{\prime}+c_{m} w_{m+1}^{\prime}\left(I_{n}-P_{m}\right)
\end{array}
$$

Proof. 1. From Algorithm 2 we can write

$$
\left[\begin{array}{llll}
x_{1} & x_{2} & \cdots & x_{m+1}
\end{array}\right]=\left[\begin{array}{llll}
v_{1} & v_{2} & \cdots & v_{m+1}
\end{array}\right]\left[\begin{array}{cccc}
\rho_{11} & \rho_{12} & \cdots & \rho_{1, m+1} \\
0 & \rho_{22} & \cdots & \rho_{2, m+1} \\
\vdots & \vdots & \ddots & \vdots \\
0 & 0 & \cdots & \rho_{m+1, m+1}
\end{array}\right]
$$

where

$$
x_{1}= \begin{cases}B, & \text { if } s_{1}=\infty \\ \left(s_{1} I_{n}-A\right)^{-1} B, & \text { if } s_{1} \neq \infty\end{cases}
$$

and for $i=2, \ldots, m$,

$$
x_{i}= \begin{cases}B, & \text { if } s_{i}=\infty \neq s_{i-1} \\ A x_{i-1}, & \text { if } s_{i}=s_{i-1}=\infty \\ \left(s_{i} I_{n}-A\right)^{-1} B, & \text { if } s_{i} \neq \infty, s_{i} \neq s_{i-1} \\ \left(s_{i} I_{n}-A\right)^{-1} x_{i-1}, & \text { if } s_{i}=s_{i-1} \neq \infty\end{cases}
$$


and

$$
x_{m+1}=A^{m_{\infty}} B .
$$

Since we assume no breakdown, $\rho_{i i} \neq 0$ for $i=1, \ldots, m$.

First, we prove the first equation in (12). Now, $x_{1}=v_{1} \rho_{11}$. Using the expressions for $x_{1}$ in (16) we have

$$
\begin{aligned}
x_{1}=\left(s_{1} I_{n}-A\right)^{-1} B & \Rightarrow A v_{1}=s_{1} v_{1}-\rho_{11}^{-1} B \\
x_{1}=B & \Rightarrow A v_{1}=\rho_{11}^{-1} A B .
\end{aligned}
$$

In either case, it follows from our construction of $v_{m+1}$ that $A v_{1} \in \operatorname{colsp}\left(V_{m+1}\right)$. Let $2<i \leq m$ and assume that

$$
A v_{j} \in \operatorname{colsp}\left(V_{m+1}\right), j=1, \ldots, i-1 .
$$

We use an induction step to prove that $A v_{i} \in \operatorname{colsp}\left(V_{m+1}\right)$. Now

$$
x_{i}=\left[\begin{array}{lll|l}
v_{1} & \ldots & v_{i-1} & v_{i}
\end{array}\right]\left[\begin{array}{c}
\rho_{1, i} \\
\vdots \\
\rho_{i-1, i} \\
\rho_{i, i}
\end{array}\right]=\left[V_{i-1} \mid v_{i}\right]\left[\begin{array}{c}
r_{\left[\frac{r_{i-1, i}}{\rho_{i, i}}\right]}^{r_{i}} \\
.
\end{array}\right.
$$

Using the expressions for $x_{i}$ in (17) we have

$$
\begin{aligned}
x_{i}=\left(s_{i} I_{n}-A\right)^{-1} B & \Rightarrow A v_{i}=\frac{s_{i}}{\rho_{i, i}} V_{i-1} r_{i-1, i}+s_{i} v_{i}-\frac{1}{\rho_{i, i}} A V_{i-1} r_{i-1, i}-\frac{1}{\rho_{i, i}} B \\
x_{i}=\left(s_{i} I_{n}-A\right)^{-1} x_{i-1} & \Rightarrow A v_{i}=\frac{s_{i}}{\rho_{i, i}} V_{i-1} r_{i-1, i}+s_{i} v_{i}-\frac{1}{\rho_{i, i}} A V_{i-1} r_{i-1, i}-\frac{1}{\rho_{i, i}} x_{i-1} \\
x_{i}=B & \Rightarrow A v_{i}=\frac{1}{\rho_{i, i}} A B-\frac{1}{\rho_{i, i}} A V_{i-1} r_{i-1, i} \\
x_{i}=A x_{i-1} & \Rightarrow A v_{i}=\frac{1}{\rho_{i, i}} A^{2} x_{i-1}-\frac{1}{\rho_{i, i}} A V_{i-1} r_{i-1, i} .
\end{aligned}
$$

It follows from (19) and the construction of $v_{m+1}$ in the lemma that $A v_{i} \in \operatorname{colsp}\left(V_{m+1}\right)$. Thus $A v_{j} \in \operatorname{colsp}\left(V_{m+1}\right)$ for $j=1, \ldots, m$. This shows that

$$
A V_{m}=V_{m} H_{m}+v_{m+1} C_{V_{m}}
$$

for some $H_{m}$ and $C_{V_{m}}$. Since $V_{m+1}$ is orthonormal, multiplying (21) from the left by $v_{m+1}^{\prime}$ gives $C_{V_{m}}=v_{m+1}^{\prime} A V_{m}$, while multiplying by $V_{m}^{\prime}$ gives $H_{m}=V_{m}^{\prime} A V_{m}$. Next, we prove the second equation in (12). Now, either $\infty \in S$, in which case $x_{j}=B$ for some $j \leq m$, or else $\infty \notin S$ in which case $x_{m+1}=B$ by our construction of $v_{m+1}$. In either case $B \in \operatorname{colsp}\left(V_{m+1}\right)$. Hence

$$
B=V_{m} L_{m}+v_{m+1} b_{m} .
$$


for some $L_{m}$ and $b_{m}$. Since $V_{m+1}$ is orthonormal, multiplying (22) from the left by $v_{m+1}^{\prime}$ gives $b_{m}=v_{m+1}^{\prime} B$, while multiplying by $V_{m}^{\prime}$ gives $L_{m}=V_{m}^{\prime} B$. Furthermore, if $\infty \in S$, so that $x_{j}=B$ for some $j \leq m$, then $b_{m}=v_{m+1}^{\prime} B=0$. Similar remarks apply to $c_{m}$.

The proof for (13) is similar and is therefore omitted.

2. The proof can be devived directly from the results of part (1). Pre-multiplying (21) by $W_{m}^{\prime}$ and rearranging gives $H_{m}=E_{m}^{-1} A_{m}-E_{m}^{-1} W_{m}^{\prime} v_{m+1} C_{V_{m}}$. Substituting $H_{m}$ in (21) gives the first equation in (14). Next, we prove the second equation in (14). Pre-multiplying (22) by $W_{m}^{\prime}$ and rearranging gives $L_{m}=E_{m}^{-1} B_{m}-E_{m}^{-1} W_{m}^{\prime} v_{m+1} b_{m}$. Substituting in (22) we get (14). The proof for (15) is similar to that for (14).

This completes the proof.

It is important to mention that Lemma 3.1 does not alter $V_{m}$ or $W_{m}$ and the only additional cost for obtaining the Arnoldi equations is an extra iteration of the Arnoldi algorithm.

Remark 3.1. The main difference between the Lanczos algorithm [17,21,26,27] and the Arnoldi process is that the orthonormality requirements $W_{m+1}^{\prime} W_{m+1}=V_{m+1}^{\prime} V_{m+1}=I_{m+1}$ are replaced by the bi-orthonormal requirement $W_{m+1}^{\prime} V_{m+1}=I_{m+1}$. Thus all the results we presented for the Arnoldi case hold for the Lanczos case as well, where now

$$
\begin{aligned}
& E_{m}=I_{m}, \quad B_{W_{m}}=W_{m}^{\prime} A v_{m+1}, \quad b_{m}=w_{m+1}^{\prime} B, \\
& w_{m+1}^{\prime} P_{m}=P_{m} v_{m+1}=0, \quad C_{V_{m}}=w_{m+1}^{\prime} A V_{m}, \quad c_{m}=C v_{m+1} \text {. }
\end{aligned}
$$

\section{Error analysis for the rational Arnoldi algorithm}

In the section we use the Arnoldi equations derived in the previous section to carry out an error analysis for the rational Arnoldi algorithm. The purpose of the error analysis is threefold. Firstly, it is useful for deriving stopping criteria for the Arnoldi algorithm. Secondly, it can be used to derive new interpolation points in an adaptive scheme. Thirdly, it is useful in any subsequent analysis, design or simulation carried out on the approximation $G_{m}(s)$.

\subsection{Residual errors}

The residual errors are useful as stopping criteria and for updating the interpolation points. With the use of the Arnoldi equations derived in Lemma 3.1, expressions for the residual errors $R_{B}(s)$ and $R_{C}(s)$, defined in (8) and (9), can be derived. Using (8), (9) and (14)

$$
\begin{aligned}
R_{B}(s) & =B-V_{m} E_{m}^{-1}\left(s E_{m}-A_{m}\right) X_{m}(s)+\left(I_{n}-P_{m}\right) v_{m+1} C_{V_{m}} X_{m}(s) \\
& =\underbrace{\left(I_{n}-P_{m}\right) v_{m+1}}_{\tilde{B}} \underbrace{\left(C_{V_{m}}\left(s E_{m}-A_{m}\right)^{-1} B_{m}+b_{m}\right)}_{\tilde{R}_{B}(s)} .
\end{aligned}
$$


Using (12) another expression can be obtained as

$$
R_{B}(s)=v_{m+1}\left(C_{V_{m}}\left(s I_{m}-H_{m}\right)^{-1} L_{m}+b_{m}\right) .
$$

Similarly a manipulation using (8), (9), (13) and (15) gives

$$
\begin{aligned}
R_{C}(s)^{\prime} & =\overbrace{\left(c_{m}+C_{m}\left(s E_{m}-A_{m}\right)^{-1} B_{W_{m}}\right)}^{\tilde{R}_{C}(s)^{\prime}} \overbrace{w_{m+1}^{\prime}\left(I_{n}-P_{m}\right)}^{\tilde{C}} \\
& =\left(K_{m}\left(s I_{m}-F_{m}\right)^{-1} B_{W_{m}}+c_{m}\right) w_{m+1}^{\prime} .
\end{aligned}
$$

These expressions are simple generalizations for the expressions for $R_{B}(s)$ and $R_{C}(s)^{\prime}$ for the standard Arnoldi process in (10) and (11). It is also straightforward to verify that the forward error expression is given by

$$
\epsilon(s):=G(s)-G_{m}(s)=\tilde{R}_{C}(s)^{\prime} \overbrace{C}^{C\left(s I_{n}-A\right)^{-1} \tilde{B}} \tilde{R}_{B}(s) .
$$

\subsection{Linear fractional transformation (LFT) uncertainty modeling}

Since the large order model $G(s)$ is to be replaced by the low order approximation $G_{m}(s)$, it is useful to regard $G_{m}(s)$ as a nominal model and $G(s)$ as an uncertain model. A very common way of relating the nominal and uncertain models is through the use of LFT uncertainty modeling [39]. In [31] the authors derived an expression of the system in terms of LFT's using matrices produced by the standard Lanczos algorithm. That was possible because of the existence of the standard Lanczos equations. In this section as an extension to the work of [31], having derived the Arnoldi equations for the rational case, LFT expressions are derived for the system using matrices produced by the two-sided rational Arnoldi algorithm. Using these representations of the system simple error expressions of the approximation will be derived for the rational Krylov methods. Let

$$
M=\left[\begin{array}{ll}
M_{11} & M_{12} \\
M_{21} & M_{22}
\end{array}\right] \in \mathcal{C}^{(p 1+p 2) \times(q 1+q 2)}
$$

and let $\Delta \in \mathcal{C}^{q 2 \times p 2}$. The lower LFT with respect to $\Delta$ is defined as $\mathcal{F}_{l}(M, \Delta):=M_{11}+M_{12} \Delta\left(I_{n}-\right.$ $\left.M_{22} \Delta\right)^{-1} M_{21}$ provided that $\left(I_{n}-M_{22} \Delta\right)^{-1}$ exists.

Theorem 4.1. Let all variables be as defined in Lemma 3.1. Then

$$
G(s)=\mathcal{F}_{l}\left(Q_{m}(s), \Delta(s)\right)
$$


where

$$
\begin{aligned}
Q_{m}(s) & \stackrel{s}{=}\left[\begin{array}{c|cc}
E_{m}^{-1} A_{m} & E_{m}^{-1} B_{m} & E_{m}^{-1} B_{W_{m}} \\
\hline C_{m} & 0 & c_{m} \\
C_{V_{m}} & b_{m} & 0
\end{array}\right]=\left[\begin{array}{cc}
G_{m}(s) & \tilde{R}_{C}(s)^{\prime} \\
\tilde{R}_{B}(s) & Q_{22}(s)
\end{array}\right] \\
\Delta(s) & \stackrel{s}{=}\left[\begin{array}{c|c}
\tilde{A} & \tilde{B} \\
\hline \tilde{C} & 0
\end{array}\right]:=\left[\begin{array}{cc|c}
A-\tilde{B} C_{V_{m}} E_{m}^{-1} W_{m}^{\prime}-V_{m} E_{m}^{-1} B_{W_{m}} \tilde{C} & \left(I_{n}-P_{m}\right) v_{m+1} \\
\hline w_{m+1}^{\prime}\left(I_{n}-P_{m}\right) & 0
\end{array}\right] .
\end{aligned}
$$

Furthermore,

$$
\epsilon(s):=G(s)-G_{m}(s) \stackrel{s}{=}\left[\begin{array}{ccc|c}
E_{m}^{-1} A_{m} & E_{m}^{-1} B_{W_{m}} \tilde{C} & 0 & E_{m}^{-1} B_{m} \\
\tilde{B} C_{V_{m}} & \tilde{A} & 0 & \tilde{B} b_{m} \\
0 & 0 & E_{m}^{-1} A_{m} & E_{m}^{-1} B_{m} \\
\hline C_{m} & c_{m} \tilde{C} & -C_{m} & 0
\end{array}\right] .
$$

Proof. It follows from Lemma 10.3 in [39] that

$$
\mathcal{F}_{l}\left(Q_{m}(s), \Delta(s)\right)=N(s)
$$

where

$$
N(s) \stackrel{s}{=}\left[\begin{array}{cc|c}
E_{m}^{-1} A_{m} & E_{m}^{-1} B_{W_{m}} \tilde{C} & E_{m}^{-1} B_{m} \\
\tilde{B} C_{V_{m}} & \tilde{A} & \tilde{B} b_{m} \\
\hline C_{m} & c_{m} \tilde{C} & 0
\end{array}\right] .
$$

Applying a similarity transformation on $N(s)$ with left and right transformation matrices given by

$$
T_{L}=\left[\begin{array}{cc}
I_{m} & -E_{m}^{-1} W_{m}^{\prime} \\
V_{m} & I_{m}-V_{m} E_{m}^{-1} W_{m}^{\prime}
\end{array}\right], \quad T_{R}=\left[\begin{array}{cc}
0 & E_{m}^{-1} W_{m}^{\prime} \\
-V_{m} & I_{m}
\end{array}\right],
$$

and using the Arnoldi equations (14) and (15) gives

$$
N(s) \stackrel{s}{=}\left[\begin{array}{cc|c}
E_{m}^{-1} A_{m} & E_{m}^{-1} B_{W_{m}} \tilde{C} & E_{m}^{-1} B_{m} \\
0 & A & B \\
\hline 0 & C & 0
\end{array}\right] \stackrel{s}{=}\left[\begin{array}{c|c}
A & B \\
\hline C & 0
\end{array}\right]=G(s) .
$$

Therefore (26) follows from (28). Finally, (27) follows from (26) and (29) by direct evaluation.

\section{$4.3 \quad$ Norm-bounded uncertainty modeling}

Another approach to modeling uncertainties is in terms of norm-bounded uncertainties on the state-space data. Since $G_{m}(s)$ is taken to be an approximation of $G(s)$, an interesting question is whether $G_{m}(s)$ is a minimal realization for a perturbed realization for $G(s)$. Using the Arnoldi equations derived in Section 3 we have the following results which relate the reduced order system data to rank one perturbations of the original data. 
Theorem 4.2. Let all variables be as defined in Lemma 3.1. Then

1. $\left(H_{m}, L_{m}\right)$ is an exact projection of the perturbed system $\left(A-\Delta_{A}, B-\Delta_{B}\right)$ where

$$
\left[\begin{array}{ll}
\Delta_{A} & \Delta_{B}
\end{array}\right]=v_{m+1}\left[\begin{array}{ll}
C_{V_{m}} & b_{m}
\end{array}\right]\left[\begin{array}{cc}
V_{m}^{\prime} & 0 \\
0 & 1
\end{array}\right] .
$$

Furthermore, $\left\|\left[\begin{array}{cc}\Delta_{A} & \Delta_{B}\end{array}\right]\right\|=\left\|\left[\begin{array}{cc}C_{V_{m}} & b_{m}\end{array}\right]\right\|$.

2. $\left(F_{m}, K_{m}\right)$ is an exact projection of the perturbed system $\left(A-\Delta_{A}, B-\Delta_{B}\right)$ where

$$
\left[\begin{array}{c}
\Delta_{A} \\
\Delta_{C}
\end{array}\right]=\left[\begin{array}{cc}
W_{m} & 0 \\
0 & 1
\end{array}\right]\left[\begin{array}{c}
B_{W_{m}} \\
c_{m}
\end{array}\right] w_{m+1}^{\prime}
$$

Furthermore, $\left\|\left[\begin{array}{cc}\Delta_{A}^{\prime} & \Delta_{C}^{\prime}\end{array}\right]\right\|=\left\|\left[\begin{array}{cc}B_{W_{m}}^{\prime} & c_{m}^{\prime}\end{array}\right]\right\|$.

3. $\left(E_{m}^{-1} A_{m}, E_{m}^{-1} B_{m}\right)$ is an exact projection of the perturbed system $\left(A-\Delta_{A}, B-\Delta_{B}\right)$ where

$$
\left[\begin{array}{ll}
\Delta_{A} & \Delta_{B}
\end{array}\right]=\left(I_{n}-P_{m}\right) v_{m+1}\left[\begin{array}{cc}
C_{V_{m}} E_{m}^{-1} & b_{m}
\end{array}\right]\left[\begin{array}{cc}
W_{m}^{\prime} & 0 \\
0 & 1
\end{array}\right] .
$$

Furthermore, $\left\|\left[\begin{array}{cc}\Delta_{A} & \Delta_{B}\end{array}\right]\right\| \leq \sqrt{\left(1+\left\|E_{m}^{-1} W_{m}^{\prime} v_{m+1}\right\|^{2}\right)}\left\|\left[\begin{array}{cc}C_{V_{m}} E_{m}^{-1} & b_{m}\end{array}\right]\right\|$.

4. $\left(A_{m} E_{m}^{-1}, C_{m} E_{m}^{-1}\right)$ is an exact projection of the perturbed system $\left(A-\Delta_{A}, C-\Delta_{C}\right)$ where

$$
\left[\begin{array}{c}
\Delta_{A} \\
\Delta_{C}
\end{array}\right]=\left[\begin{array}{cc}
V_{m} & 0 \\
0 & 1
\end{array}\right]\left[\begin{array}{c}
E_{m}^{-1} B_{W_{m}} \\
c_{m}
\end{array}\right] w_{m+1}^{\prime}\left(I_{n}-P_{m}\right) .
$$

Furthermore, $\left\|\left[\begin{array}{cc}\tilde{\Delta}_{A}^{\prime} & \tilde{\Delta}_{C}^{\prime}\end{array}\right]\right\| \leq \sqrt{\left(1+\left\|\left(E_{m}^{\prime}\right)^{-1} V_{m}^{\prime} w_{m+1}\right\|^{2}\right)}\left\|\left[\begin{array}{ll}\left(E_{m}^{-1} B_{W_{m}}\right)^{\prime} & c_{m}^{\prime}\end{array}\right]\right\|$.

Proof. The results in 1. and 2. follow by rewriting (12) and (13) as

$$
\begin{aligned}
& V_{m} H_{m}=\left(A-v_{m+1} C_{V_{m}} V_{m}^{\prime}\right) V_{m}, \quad V_{m} L_{m}=B-v_{m+1} b_{m}, \\
& F_{m} W_{m}^{\prime}=W_{m}^{\prime}\left(A-W_{m} B_{W_{m}} w_{m+1}^{\prime}\right), \quad K_{m} W_{m}^{\prime}=C-c_{m} w_{m+1}^{\prime} \text {, }
\end{aligned}
$$

respectively, using $W_{m+1}^{\prime} W_{m+1}=V_{m+1}^{\prime} V_{m+1}=I_{m+1}$. Since $V_{m+1}$ and $W_{m+1}$ are orthonormal, it is clear that the norm of the perturbation of the perturbed systems are as given in the theorem.

The results in 3 . and 4 . follow by rewriting (14) and (15) as

$$
\begin{aligned}
& V_{m} E_{m}^{-1} A_{m}=\left(A-\left(I_{n}-P_{m}\right) v_{m+1} C_{V_{m}} E_{m}^{-1} W_{m}^{\prime}\right) V_{m}, \quad V_{m} E_{m}^{-1} B_{m}=B-\left(I_{n}-P_{m}\right) v_{m+1} b_{m}, \\
& A_{m} E_{m}^{-1} W_{m}^{\prime}=W_{m}^{\prime}\left(A-V_{m} E_{m}^{-1} B_{W_{m}} w_{m+1}^{\prime}\left(I_{n}-P_{m}\right)\right), \quad C_{m} E_{m}^{-1} W_{m}^{\prime}=C-c_{m} w_{m+1}^{\prime}\left(I_{n}-P_{m}\right),
\end{aligned}
$$

respectively, using $W_{m+1}^{\prime} W_{m+1}=V_{m+1}^{\prime} V_{m+1}=I_{m+1}, E_{m}=W_{m}^{\prime} V_{m}$ and $P_{m}=V_{m} E_{m}^{-1} W_{m}^{\prime}$. Using $V_{m}^{\prime} V_{m}=W_{m}^{\prime} W_{m}=I_{m}$ and $P_{m}=V_{m} E_{m}^{-1} W_{m}^{\prime}$ we can also prove the bound on the 
perturbation of the system in case 3. as follows.

$$
\begin{aligned}
\left\|\left[\begin{array}{ll}
\Delta_{A} & \Delta_{B}
\end{array}\right]\right\| & \leq\left\|\left(I_{n}-P_{m}\right) v_{m+1}\right\|\left\|\left[\begin{array}{ll}
C_{V_{m}} E_{m}^{-1} & b_{m}
\end{array}\right]\right\| \\
& =\sqrt{v_{m+1}^{\prime}\left(I_{n}-P_{m}^{\prime}\right)\left(I_{n}-P_{m}\right) v_{m+1}}\left\|\left[\begin{array}{ll}
C_{V_{m}} E_{m}^{-1} & b_{m}
\end{array}\right]\right\| \\
& =\sqrt{\left(1+v_{m+1}^{\prime} P_{m}^{\prime} P_{m} v_{m+1}\right)}\left\|\left[\begin{array}{cc}
C_{V_{m}} E_{m}^{-1} & b_{m}
\end{array}\right]\right\| \\
& =\sqrt{\left(1+\left\|E_{m}^{-1} W_{m}^{\prime} v_{m+1}\right\|^{2}\right)}\left\|\left[\begin{array}{ll}
C_{V_{m}} E_{m}^{-1} & b_{m}
\end{array}\right]\right\| .
\end{aligned}
$$

The bound on the norm of perturbation of the system in case 4. can be obtained by following similar steps.

\section{Adaptive schemes for the rational methods}

The problem in adaptive rational methods is to decide on the next interpolation point on every iteration. The interpolation point selection problem, for example, often appears in cases where simulation of large scale circuits is required. In [11] the authors use the Arnoldi algorithm to produce a number of interpolating approximations of the original system around single expansion points and using the resulting approximations they compute approximations of some poles and residues of the system. They suggest to use a subset of the approximated poles as interpolation points. Another interesting method for interpolation point selection in the area of circuits and systems is related to series expansions based on orthonormal polynomials, such as Chebyshev polynomials, and to series expansions based on generalised orthonormal basis functions in Hilbert and Hardy spaces [38].

The adaptive method presented in this paper uses a different approach and is more related to the approaches used in the following. Grimme in his thesis [20] explains the interpolation point selection problem in terms of approximating the eigenvalues of the original system. According to his observations, Grimme suggested using a combination of real and imaginary interpolation points which are logarithmically or linearly spaced on the real and imaginary axis. Given a sufficient number of interpolation points one can obtain reduced models that are good approximations of the original system. However the appropriate number of points cannot be known a priori. In his thesis he also suggested a method for determining the order and the choice of the imaginary interpolation points adaptively on every iteration of the rational Krylov algorithms. The suggested approach tends to reduce the $\mathcal{H}_{\infty}$ norm of the transfer function of the residual errors or the $\mathcal{H}_{\infty}$ norm of the transfer function of an error estimate of the approximation at each step. Error estimation expressions and residual error expressions have also been derived in $[5,17,21]$. The authors in $[10,17,28]$ use the same adaptive approach to choose the next interpolation frequency amongst a fixed set of interpolation points by using expressions related 
to the moments errors and [13] chooses a single interpolation point in the frequency range of interest. The material presented in this section addresses similar issues as above through the use of Arnoldi-like equations for the rational case.

The adaptive rational Arnoldi procedure given in Algorithm 3 below computes the bases $V_{m}$ and $W_{m}$ of the union of Krylov subspaces as defined in Theorem 2.1 where the interpolation points $s_{k} \in S$ and $\tilde{s}_{k} \in \tilde{S}$ are computed adaptively; on every iteration the next interpolation points are computed to be the frequencies at which the $\mathcal{H}_{\infty}$ norm of an error expression is achieved. For simplicity of presentation it is assumed that $m_{s_{k}}=m_{\tilde{s}_{k}}=1$ and that $s_{i} \neq s_{j}$ and $\tilde{s}_{i} \neq \tilde{s}_{j}$ for $i \neq j$. One could choose to perform multiple iterations before selecting a new interpolation point by setting the multiplicities $m_{s_{k}}=m_{\tilde{s}_{k}}>1$. A number of different options for the error expression to be computed in line 28 can be chosen depending on the available computational power or time constraints.

Exact expressions for the forward error were derived in (25) and (27). Evaluating these expressions on every iteration is not efficient since they involve terms related to the large scale system. The residual errors $R_{B}\left(X_{m}\right)$ and $R_{C}\left(Y_{m}\right)$ on the other hand involve terms related to the reduced system only and their computation on every iteration is feasible. Even though the computation of the residual errors may not be a good approximation of the forward error, it is a common observation that small residual errors at a given frequency imply small forward error at that frequency [20]. This makes the residual error expressions a good choice to minimise on every iteration of the Krylov algorithms. For even less computational effort one could use just the frequency dependent terms of the residual errors defined in (23) and (24).

For the forward error expressions defined in (25) and (27) to involve only reduced order terms one could use their approximations as

$$
\hat{\epsilon}_{H_{r}}(s)=\tilde{R}_{C}^{\prime}(s) H_{r}(s) \tilde{R}_{B}(s)
$$

and

$$
\hat{\epsilon}_{\Delta_{r}}(s) \stackrel{s}{=}\left[\begin{array}{ccc|c}
E_{m}^{-1} A_{m} & E_{m}^{-1} B_{W_{m}} \tilde{C}_{r} & 0 & E_{m}^{-1} B_{m} \\
\tilde{B}_{r} C_{V_{m}} & \tilde{A}_{r} & 0 & \tilde{B}_{r} b_{m} \\
0 & 0 & E_{m}^{-1} A_{m} & E_{m}^{-1} B_{m} \\
\hline C_{m} & c_{m} \tilde{C}_{r} & -C_{m} & 0
\end{array}\right]
$$

where $H_{r}(s)$ is an approximation of $H(s) \stackrel{s}{=}(A, \tilde{B}, \tilde{C}, 0)$ defined in $(25)$ by the projection

$$
H_{r}(s) \stackrel{s}{=}\left(\left(\tilde{W}^{\prime} \tilde{V}\right)^{-1} \tilde{W}^{\prime} A \tilde{V},\left(\tilde{W}^{\prime} \tilde{V}\right)^{-1} \tilde{W} \tilde{W}^{\prime} \tilde{B}, \tilde{C} \tilde{V}, 0\right)
$$

and the triple $\left(\tilde{A}_{r}, \tilde{B}_{r}, \tilde{C}_{r}\right)$ is obtained by an approximation $\Delta_{r}(s)$ of $\Delta(s) \stackrel{s}{=}(\tilde{A}, \tilde{B}, \tilde{C}, 0)$ defined 


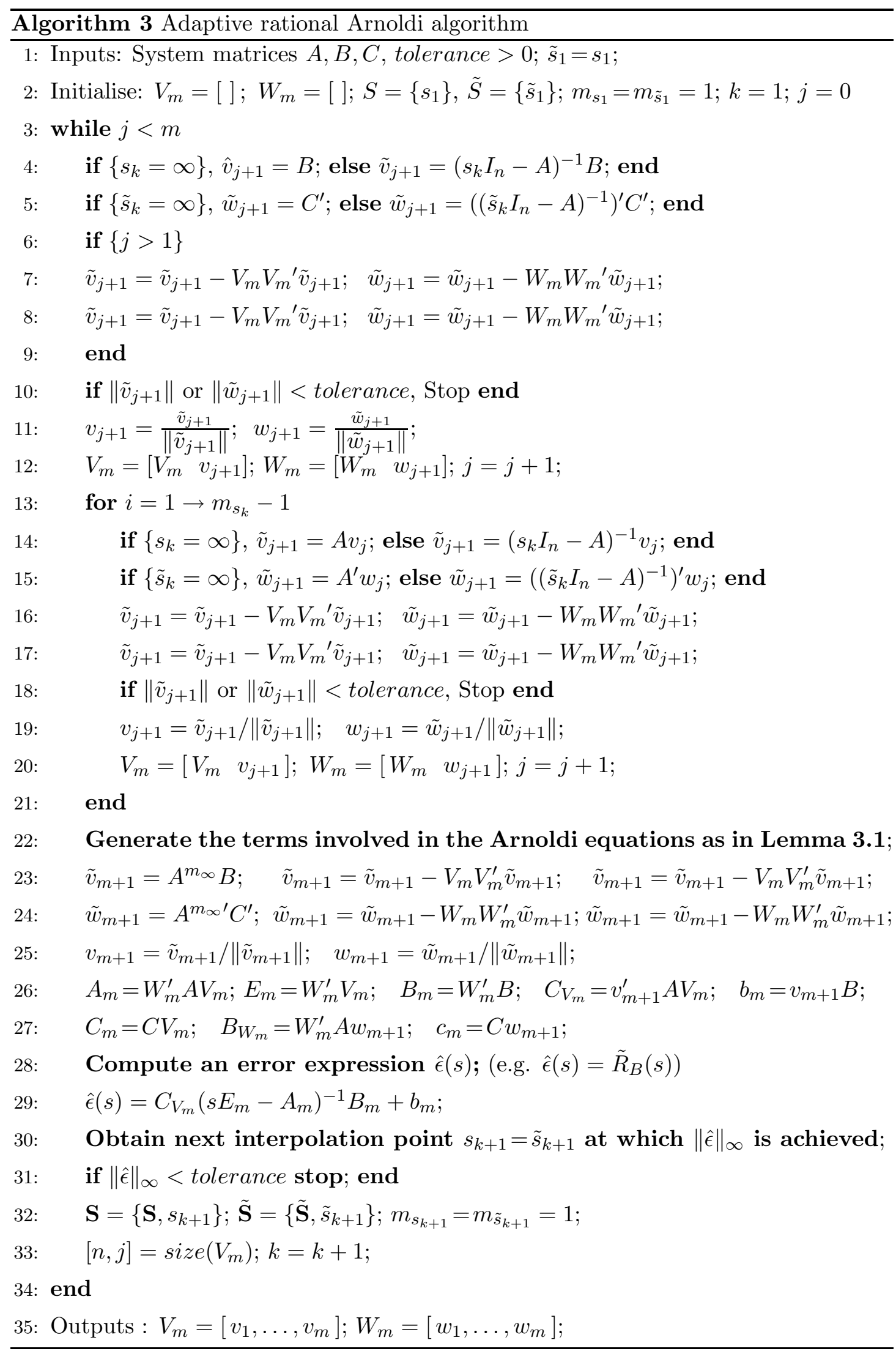


in Theorem 4.1 by the projection

$$
\Delta_{r}(s) \stackrel{s}{=}(\underbrace{\left(\tilde{W}^{\prime} \tilde{V}\right)^{-1} \tilde{W}^{\prime} \tilde{A} \tilde{V}}_{\tilde{A}_{r}}, \underbrace{\left(\tilde{W}^{\prime} \tilde{V}\right)^{-1} \tilde{W}{ }^{\prime} \tilde{B}}_{\tilde{B}_{r}}, \underbrace{\tilde{C} \tilde{V}}_{\tilde{C}_{r}}, 0)
$$

assuming $\left(\tilde{W}^{\prime} \tilde{V}\right)$ is non-singular. The bases $\tilde{V}$ and $\tilde{W}$ are constructed by the rational Arnoldi algorithm given in Algorithm 2. Note that the order of the forward error estimates is $2 m+r<n$ where $r$ is the order of approximation of $H(s)$ or $\Delta(s)$. Different methods of approximation of $H(s)$ and $\Delta(s)$ depending on the available computational power and the time constrains result in different error estimates and therefore different adaptive schemes.

Remark 5.1. A full theoretical analysis of the computation complexity involved in the proposed adaptive methods is out of the scope of this paper. However we note that all the complex computations are at level $m$ and can be considered as low cost operations.

A list of suggestions for the error estimates $\hat{\epsilon}(s)$ to be used in line 28 of Algorithm 3 is given below in Table 1.

\section{$6 \quad$ Numerical Examples}

In this section the evaluation and comparison of the methods suggested in this paper is obtained by applying model reduction on two stable models whose singular values plots are given in Figure 1 below. Model rnd500 is a random model of order $n=500$ generated by the rss function in MATLAB [29], while 1riis11 is a model of order $n=270$ with transfer function being equal to the $(1,1)$ transfer function of the component $1 r$ of the International Space Station and is frequently used as a benchmark for model reduction of large scale systems [1,9]. Note from the frequency response plots in Figure 1, that both models exhibit complex dynamics and are therefore difficult to approximate. 
Table 1: Error Estimates

\begin{tabular}{|c|c|}
\hline Error estimate $\hat{\epsilon}(s)$ & Description \\
\hline$\hat{\epsilon}_{R_{B}}, \hat{\epsilon}_{R_{C}}$ & $\begin{array}{l}\text { These are the frequency dependent parts of the } \\
\text { residual errors defined in }(23) \text { and }(24) \text {. }\end{array}$ \\
\hline$\hat{\epsilon}_{H_{1}^{\text {single }}}, \hat{\epsilon}_{\Delta_{1}^{\text {single }}}$ & $\begin{array}{l}\text { These are the forward error estimations defined in } \\
(30) \text { and }(31) \text { where } H_{1}^{\text {single }}(s) \text { or } \Delta_{1}^{\text {single }}(s) \text { are } \\
\text { obtained by single point interpolation at the last } \\
\text { points added in } S \text { and } \tilde{S} \text { on the given iteration. }\end{array}$ \\
\hline$\hat{\epsilon}_{H_{m+1}^{\text {extended }}}, \hat{\epsilon}_{\Delta_{m+1}^{\text {extended }}}$ & $\begin{array}{l}\text { These are the forward error estimations defined in } \\
(30) \text { and }(31) \text { where } H_{m+1}^{\text {extended }}(s) \text { or } \Delta_{m+1}^{\text {extended }}(s) \text { are } \\
\text { extended versions of single point interpolations ob- } \\
\text { tained by projection with the bases } \tilde{V}=\left[V_{m}, \hat{v}\right] \in \\
\mathbb{C}^{n \times m+1} \text { and } \tilde{W}=\left[W_{m}, \hat{w}\right] \in \mathbb{C}^{n \times m+1} \text { where } \hat{v}, \hat{w} \\
\text { are the columns obtained by single interpolation as } \\
\text { described above and orthogonalised with respect to } \\
\text { the bases } V_{m} \text { and } W_{m} \text { constructed on the given it- } \\
\text { eration. }\end{array}$ \\
\hline$\hat{\epsilon}_{H_{k}^{\text {rational }}}, \hat{\epsilon}_{\Delta_{k}^{\text {rational }}}$ & $\begin{array}{l}\text { These are the forward error estimations defined in } \\
(30) \text { and }(31) \text { where } H_{k}^{\text {rational }}(s) \text { or } \Delta_{k}^{\text {rational }}(s) \text { are } \\
\text { obtained by rational interpolation at the points in } \\
S \text { and } \tilde{S} \text {. }\end{array}$ \\
\hline
\end{tabular}



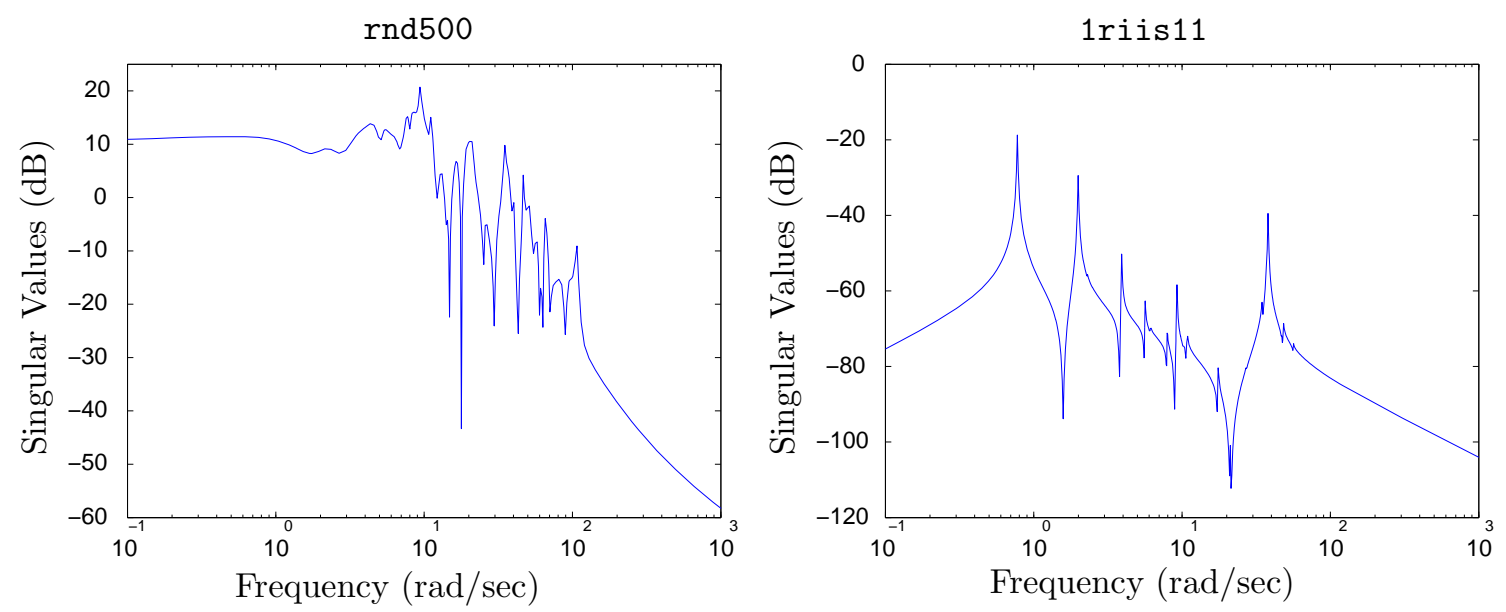

Figure 1: Singular values plots for the models rnd500 and 1riis11

To compare the performance of the adaptive algorithm using the various error estimates given in Table 1, Algorithm 3 was used for each one of them. To measure the accuracy of the approximations, in each case the relative $\mathcal{H}_{\infty}$ error which is defined as $\mathcal{E}_{\infty}:=\frac{\left\|G-G_{m}\right\|_{\infty}}{\|G\|_{\infty}}$, was computed for different values of $m$ and the results are shown below, in Figure 2. The results are also compared with the performance of balanced truncation and to the results of rational Arnoldi method interpolating at random interpolation points on the imaginary axis.

Observing part (a) of Figure 2 it is evident that a careful selection of interpolation points using information from the residual errors produces greatly improved approximations with lower relative error compared to the approximations obtained by the rational Arnoldi method at randomly selected interpolation points. As expected in all the cases the relative error tends to decrease as the order of approximation increases and the relative error tends to decrease faster when the interpolation points are computed using more accurate error estimates. Observing part (c) of the same figure it can be noticed that the error estimate $\hat{\epsilon}_{\Delta_{1}^{\text {single }}}$ which is of order $2 m+1$ seems to possess nearly identical information about the real error expression as the error estimate $\hat{\epsilon}_{\Delta_{m+1}^{\text {extended }}}$ which is of order $3 m+1$.

Generally it can be said that $\hat{\epsilon}_{H_{m}^{\text {rational }}}$ and $\hat{\epsilon}_{\Delta_{m}^{\text {rational }}}$ are equivalent error estimates. They provide the most accurate information for the interpolation points to be used but they also require more computation time for their construction. The estimates $\hat{\epsilon}_{H_{m+1}^{\text {extended }}}, \hat{\epsilon}_{\Delta_{m+1}^{\text {extended }}}$ and $\hat{\epsilon}_{\Delta_{1}^{\text {single }}}$ can also be considered as equivalent error estimates. These estimates are improved considerably when the order of approximation is increased. Moreover $\hat{\epsilon}_{\Delta_{1}^{\text {single }}}$ is less expensive to compute and it is therefore preferred over the other two. Finally the other estimates are less accurate but their approximation improves as the order of approximation increases.

Therefore for accurate interpolation point selection with minimum computational effort the 

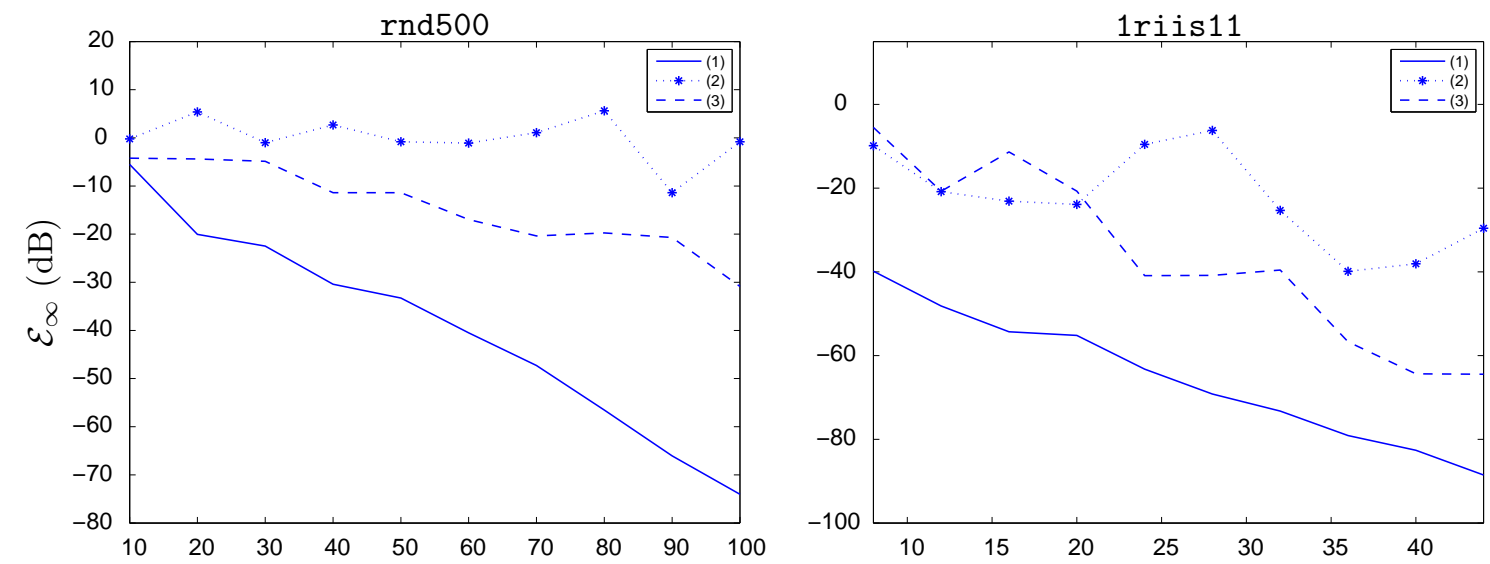

(a) : Balanced Truncation (1), Rational Arnoldi (2), Adaptive Arnoldi with $\hat{\epsilon}_{\mathcal{R}_{B}}, \hat{\epsilon}_{\mathcal{R}_{C}}$ (3).
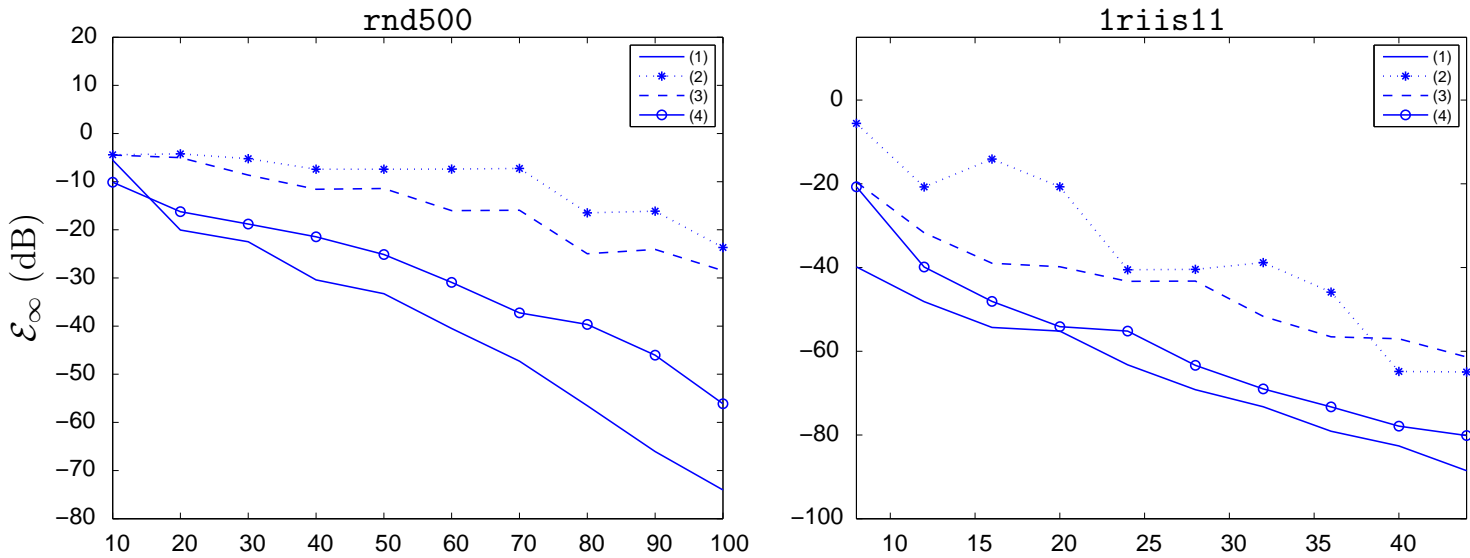

(b) : Balanced Truncation (1), Adaptive Arnoldi with $\hat{\epsilon}_{H_{1}^{\text {single }}}(2), \hat{\epsilon}_{H_{m+1}^{\text {extended }}}(3), \hat{\epsilon}_{H_{m}^{\text {rational }}}(4)$.
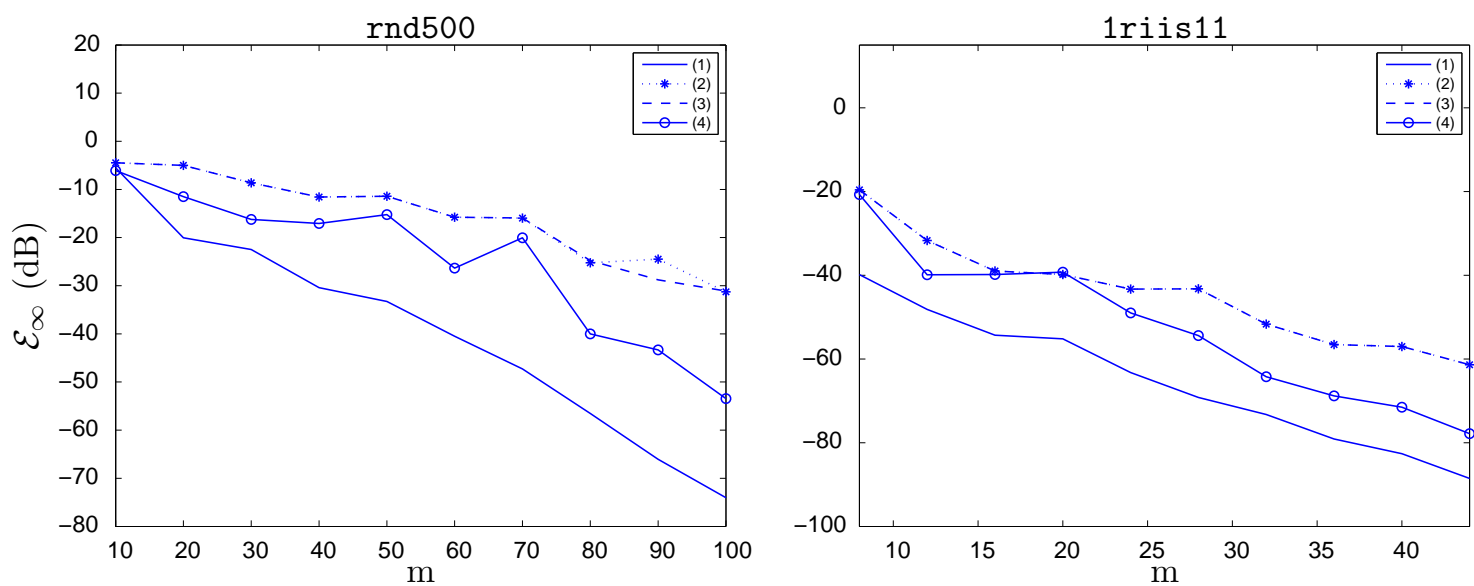

(c) : Balanced Truncation (1), Adaptive Arnoldi with $\hat{\epsilon}_{\Delta_{1}^{\text {single }}}(2), \hat{\epsilon}_{\Delta_{m+1}^{\text {extended }}}(3), \hat{\epsilon}_{\Delta_{m}^{\text {rational }}}$ (4).

Figure 2: Relative $\mathcal{H}_{\infty}$ error plots for the adaptive Arnoldi schemes 
adaptive Arnoldi algorithm given in Algorithm 3 could be implemented to choose in line 28 a more accurate and expensive estimate while the order of approximation remains low and then switch to a less expensive estimate to avoid intensive computations.

\section{Conclusions}

The work of this paper develops Arnoldi-like and Lanczos-like equations for the rational case in the standard form. The main additional cost for deriving these simple expressions is an extra iteration of the algorithms which does not require the computation of the inverse of any matrix; due to the low cost the methods can be applied efficiently for the approximation of large scale systems. Using the Arnoldi and Lanczos-like equations simple residual error expressions and forward error approximations have also been developed. The residual error expressions as well as the approximations of the forward error expressions derived, contain reduced terms only and can be computed efficiently on every iteration providing useful information about the selection of the interpolation frequencies at which the moments should be matched. The adaptive method developed tends to reduce the error in the range of frequencies where there is useful information without a priori knowledge of the original system and improves greatly the approximations obtained by rational Arnoldi methods.

Thus we suggest to use the more expensive $\hat{\epsilon}(s)$ when time constraint is not the main concern or a less expensive $\hat{\epsilon}(s)$ for faster computation. Alternatively a combination of the interpolation point selection methods could be used such that an expensive, and hence more accurate, method can be used while the order of the system remains small and a less expensive method could be used when the order $m$ becomes larger.

\section{References}

[1] A.C. Antoulas. Short course on model reduction of large-scale dynamical systems. Technical report, Rise University, Houston, TX., 2001.

[2] A.C. Antoulas. Lectures on the Approximation of Large-Scale Dynamical Systems. SIAM, 2005.

[3] A.C. Antoulas, D.C. Sorensen, and S. Gugercin. A survey of model reduction methods for large-scale systems. Contemp. Math, 280:193-219, 2001.

[4] W.E. Arnoldi. The principle of minimized iterations in the solution of matrix eigenvalue problem. Quart. Appl. Math., 9:17-29, 1951. 
[5] Z. Bai and Q. Ye. Error estimation of the padé approximation of transfer functions via the lanczos process. Electronic transactions on Numerical Analysis, 7:1-17, 1998.

[6] D.L. Boley. Krylov subspace methods in linear control and model reduction: A survey. In Cornelius Lanczos Centenary Conference, pages 377-379, December 1993.

[7] D.L. Boley and G. Golub. The nonsymmetric Lanczos algorithm and controllability. Syst. Contr. Lett., 16:97-105, 1991.

[8] A. Bultheel and M. Van Barel. Padé techniques for model reduction in linear system theory: a survey. J. Comput. Appl. Math, 14:401-438, March 1986.

[9] Y. Chahlaoui and P.V. Dooren. A collection of benchmark examples for model reduction of linear time invariant dynamical systems. Technical report, February 2002.

[10] C.C. Chu and H.J. Lee. Applications of multi-point arnoldi algorithms to linear lumbed transformer model simplifications. In IEEE, Power Engineering Society Summer Meeting, volume 4, pages 2406-2411, 2000.

[11] J. Cullum, A. Ruehli, and T. Zhang. A method for reduced-order modeling and simulation of large interconnect circuits and its application to PEEC models with retardation. IEEE Trans. on Circuits and Systems-II: Analog and Digital signal processing, 47(4):261-273, April 2000.

[12] J. Cullum and T. Zhang. Two-sided Arnoldi and nonsymmetric Lanczos algorithms. SIAM J. Matrix Anal. Appl, 24(2):303-319, 2002.

[13] P. Feldman and R.W. Freund. Efficient linear circuit analysis by Padé approximations via the Lanczos method. IEEE Trans. Computed-Aided Design of integrated circuits and systems, 14(5):639-649, May 1995.

[14] M. Frangos and I.M. Jaimoukha. Adaptive rational Krylov algorithms for model reduction. In Proc. European Control Conference, pages 4179-4186, July 2007.

[15] M. Frangos and I.M. Jaimoukha. Rational interpolation: Modified rational Arnoldi algorithm and Arnoldi-like equations. In Proc. IEEE Conference on Decision and Control, pages 4379-4384, December 2007.

[16] K. Gallivan, E. Grimme, and P. Van Dooren. Padé approximation of large-scale dynamic systems with Lanczos methods. In Proc. IEEE Conference on Decision and Control, 1994.

[17] K. Gallivan, E. Grimme, and P. Van Dooren. A rational Lanczos algorithm for model reduction. Numer. Algorithms, 12:33-64, 1996. 
[18] K. Gallivan, A. Vandendorpe, and P. Van Dooren. Sylvester equations and projection-based model reduction. J. Comput. Appl. Math, 162:213-229, 2004.

[19] W.B. Gragg and A. Lindquist. On the partial realization problem. Linear Algebra and its Applications, 50:277-319, 1983.

[20] E. Grimme. Krylov projection methods for model reduction. PhD thesis, University of Illinois at Urbana-Champain, Urbana, Illinois, 1997.

[21] E. Grimme, K. Gallivan, and P. Van Dooren. A rational Lanczos algorithm for model reduction II: Interpolation point selection. Technical report, University of Illinois at Urbana Champaign., 1998.

[22] E.J. Grimme, D.C. Sorensen, and P. Van Dooren. Model reduction of state space systems via an implicitly restarted Lanczos method. Numer. Algorithms, 12:1-31, 1995.

[23] I.M. Jaimoukha and E.M. Kasenally. Krylov subspace methods for solving large lyapunov equations. SIAM J. Matrix Anal. Appl, 31(1):227-251, 1994.

[24] I.M. Jaimoukha and E.M. Kasenally. Oblique projection methods for large-scale model reduction. SIAM J. Matrix Anal. Appl, 16(2):602-627, 1995.

[25] I.M. Jaimoukha and E.M. Kasenally. Implicitly restarted Krylov subspace methods for stable partial realizations. SIMAX, 18(3):633-652, 1997.

[26] C. Lanczos. An iteration method for the solution of the eigenvalue problem of linear differential and integral operators. J. Res. Nat. Bur. Stand, 45:255-282, 1950.

[27] C. Lanczos. Solution of systems of linear equations by minimized iterations. J. Res. Nat. Bur. Stand, 49:33-53, 1952.

[28] H.J. Lee, C.C. Chu, and W.S. Feng. An adaptive-order rational Arnoldi method for modelorder reductions of linear time-invariant systems. Linear Algebra and its Applications, 415:235-261, 2006.

[29] Matlab. The MathWorks, Inc., Copyright 1994-2008.

[30] V. Papakos. Restarted Lanczos algorithms for model reduction. PhD thesis, Imperial College of Science, Technology and Medicine, London,UK, Mar 2003.

[31] V. Papakos and I.M. Jaimoukha. Model reduction via an LFT-based explicitly restarted nonsymmetric Lanczos algorithm. In MTNS-02, 2002.

[32] A. Ruhe. The rational Krylov algorithm for nonsymmetric eigenvalue problems III: complex shifts for real matrices. BIT Numerical Mathematics, 34:165-176, November 1994. 
[33] A. Ruhe. Rational Krylov algorithms for nonsymmetric eigenvalue problems, II: Matrix pairs. Linear Algebra and its Applications, 197-198:283-295, January-February 1994.

[34] A. Ruhe. Rational Krylov: A practical algorithm for large sparse nonsymmetric matrix pencils. SIAM J. Sci. Comput., 19:1535-1551, September 1998.

[35] Y. Saad. Analysis on some Krylov subspace approximations to the matrix exponential operator. SIAM J. Numer. Anal., 29:209-228, 1992.

[36] Y. Saad and Henk A. van der Vorst. Iterative solution of linear systems in the 20th century. J. Comput. Appl. Math, 66:1-33, 2000.

[37] D. Sorensen. Implicit application of polynomial filters in a k-step Arnoldi method. SIAM J. Matrix Anal. Appl, 13(1):357-385, 1992.

[38] L.M. Wang, C.C. Chu, Q. Yu, and E.S. Kuh. On projection-based algorithms for model order reduction of interconnects. IEEE Trans. on Circuits and Systems-I: Fundamental Theory and Applications, 49(11):1563-1585, November 2002.

[39] K. Zhou, J.C. Doyle, and K. Glover. Robust and Optimal Control. Prentice Hall, 1995. 